\title{
Impactos de la Implementación del Corredor Vial Bioceánico en la Educación escolar en la frontera Brasil/Paraguay: conociendo el contexto
}

\author{
Impactos da Implementação do Corredor Rodoviário Bioceânico na educação \\ escolar na fronteira Brasil/Paraguai: conhecendo o contexto \\ Impacts of the Implementation of the Bioceanic Road Corridor on School \\ education in the Brazil/Paraguay border: knowing the context
}

\author{
Jaqueline Daniela Basso ${ }^{1}$ \\ Vanessa Arlésia de Souza Ferretti ${ }^{1}$
}

Recibido el 30 de jun. de 2021; revisado e aprobado el 17 sept. de 2021; aceptado el 13 oct. de 2021 DOI: http://dx.doi.org/10.20435/inter.v22i4.3423

\begin{abstract}
Resumen: A pesar de sus méritos eminentemente económicos, regionales y geopolíticos, la implementación del Corredor Bioceánico: Ruta Porto Murtinho-Puertos del Norte de Chile también requiere de la inteligibilidad de sus diversos impactos sociales en las comunidades locales, incluidas las que afectan la educación pública. En este sentido, este trabajo tiene como objetivo comprender el contexto social, económico y educativo del municipio de Mato Grosso do Sul de Porto Murtinho, que limita con Carmelo Peralta en Paraguay y es el punto de partida de la Ruta en Brasil. Por ello, realizamos una investigación bibliográfica y estadística, mediante la recopilación y análisis de indicadores oficiales generados por el Instituto Brasileiro de Geografia e Estatística (IBGE) y el Instituto Nacional de Estudos e Pesquisas Educacionais Anísio Teixeira (INEP). Los resultados identificaron que Porto Murtinho es un municipio pequeño, con una economía basada principalmente en la agricultura, las redes de educación pública atienden a poco más de cuatro mil estudiantes diariamente. Una demanda que está marcada por la diversidad cultural y lingüística dada la presencia de extranjeros y también de poblaciones indígenas. Por lo tanto, creemos que la implementación del Corredor Bioceánico se enfocará en un contexto que en sí mismo es diverso en términos culturales y lingüísticos y tenderá a incrementar la demanda de educación escolar ya que puede impulsar la permanencia de habitantes que actualmente no encuentran trabajo en el municipio y también la llegada de nuevos sujetos, dado el potencial crecimiento económico que representa la implementación del Corredor Bioceánico.
\end{abstract}

Palabras clave: Corredor Vial Bioceánico; Porto Murtinho; impactos sociales; enseñanza.

Resumo: A despeito dos méritos eminentemente econômicos, a implementação do Corredor Bioceânico: Rota Porto Murtinho- Portos do Norte do Chile requer também a inteligibilidade de seus diversos impactos sociais sobre as comunidades locais, dentre eles, aqueles que incidem sobre a educação pública. Nesse sentido, o presente trabalho tem por objetivo compreender o contexto social, econômico e, especialmente, educacional do município sul mato-grossense de Porto Murtinho, que faz fronteira com Carmelo Peralta no Paraguai e é o ponto de saída da Rota no Brasil. Para tanto, realizamos pesquisa de cunho bibliográfico e estatístico, por meio da coleta e análise de indicadores oficiais gerados pelo Instituto Brasileiro de Geografia e Estatística (IBGE) e Instituto Nacional de Estudos e Pesquisas Educacionais Anísio Teixeira (INEP).Os resultados mostram que Porto Murtinho, um município de pequeno porte, tem sua economia assentada prioritariamente na agropecuária; a rede pública de ensino atende pouco mais de quatro mil alunos diariamente e essa demanda é marcada pela diversidade cultural e linguística dada a presença de estrangeiros e também de populações indígenas. A partir desses dados, portanto, percebe-se que a implementação do Corredor Bioceânico incidirá sobre um contexto por si só já diverso e tenderá a ampliar a demanda por educação escolar à medida que deve impulsionar a permanência de habitantes que hoje não encontram postos de trabalho no município, e também a chegada de novos sujeitos, dado o potencial crescimento econômico representado pela implementação do Corredor Bioceânico. Assim, a implementação deverá vir acompanhada de investimentos na educação, objetivando sanar lacunas que já existiam e atender as necessidades emergentes.

Palavras-chave: Corredor Rodoviário Bioceânico; Porto Murtinho; impactos sociais; educação escolar.

Abstract: Despite its eminently economic merits, the implementation of the Bioceanic Corridor: Porto Murtinho-Portos do Norte Chile Route also requires the intelligibility of its various social impacts on local communities, including those that affect public education. In this sense, this work aims to understand the social, economic and, especially, educational context of the southern Mato Grosso municipality of Porto

${ }^{1}$ Universidade Estadual de Mato Grosso do Sul, Campo Grande, Mato Grosso do Sul, Brasil. 
Murtinho, which borders Carmelo Peralta in Paraguay and is the starting point of the Route in Brazil. Therefore, we carried out bibliographical and statistical research, through the collection and analysis of official indicators generated by Instituto Brasileiro de Geografia e Estatística (IBGE) and Instituto Nacional de Estudos e Pesquisas Educacionais Anísio Teixeira (INEP). The results show that Porto Murtinho, a small town, has its economy based primarily on agriculture; the public school system serves just over four thousand students daily and this demand is marked by cultural and linguistic diversity given the presence of foreigners and indigenous populations as well. Based on these data, therefore, it is clear that the implementation of the Bioceanic Corridor will focus on a context in itself that is already diverse and will tend to increase the demand for school education as it should boost the permanence of inhabitants who currently do not find jobs in the municipality, and also the arrival of new subjects, given the potential economic growth represented by the implementation of the Bioceanic Corridor. Thus, the implementation should be accompanied by investments in education, aiming to fill gaps that already existed and meet emerging needs.

Keywords: Bioceanic Highway Corridor; Porto Murtinho; social impacts; schooling.

\section{CONSIDERACIONES INICIALES}

A pesar de sus méritos eminentemente económicos, en términos de economía regional y geopolítica; la implementación del Corredor Bioceánico: Ruta Porto Murtinho - Puertos del Norte de Chile también requiere la inteligibilidad de sus diversos impactos sociales en las comunidades locales. En este sentido, se pueden destacar, por ejemplo, los efectos del proyecto sobre el contexto educativo. Cabe mencionar aquí el aumento repentino de la población estacional y, con él, la demanda de más escuela(s); la facilitación del tránsito y el intercambio cultural; mayor integración universitaria y movilidad académica; la implementación de políticas educativas para el plurilingüismo, entre otros. Tales efectos muestran la relevancia de la planificación para minimizar los impactos negativos y potenciar aspectos que apuntan al desarrollo socioeconómico y cultural local, especialmente desde la educación. Esta planificación, a su vez, exige un relevamiento y sistematización de la información relevante al contexto en cuestión. Ese es precisamente el objetivo de este enfoque.

En resumen, busca describir el contexto local en cuanto a indicadores poblacionales, económicos y educativos. Para ello, se realiza una descripción y análisis cualitativo de los indicadores estadísticos generados por el Instituto Brasileño de Geografía y Estadística (IBGE) y el Instituto Nacional de Investigaciones y Estudios Educativos Anísio Teixeira (INEP) a través de Censos Demográficos y Educativos en los años de 2019 y 2020 y el Catálogo de Escuelas 2020. El análisis cualitativo se refiere, sobre todo, al hecho de que los datos son "interpretados y contextualizados a la luz de la dinámica social más amplia”, como explica Gamboa (2009, p. 106), en soporte teórico asumido en esta investigación, de acuerdo con la revisión de la literatura presentada.

Así, seguimos con I) una breve revisión histórica de la implementación del Corredor Vial Bioceânico, para luego II) describir y III) analizar algunos indicadores poblacionales, económicos y educativos del municipio de Porto Murtinho, con el fin de identificar la dimensión de la red de educación pública y, finalmente, problematizar las condiciones de escolarización en el contexto fronterizo.

\section{EL CORREDOR BIOCEÁNICO Y LA CONSTITUCIÓN DE UNIRILA: CONTEXTUALIZANDO ESTA INVESTIGACIÓN}

El Corredor Vial Bioceánico: Ruta Porto Murtinho - Puertos del Norte de Chile no es una iniciativa aislada en América Latina, ya se han diseñado otras rutas que están posibilitando el flujo de bienes y el flujo cultural y social en este territorio. Oliveira (2010) destaca que comúnmente 
se perciben contradicciones entre los proyectos de Rutas y las poblaciones de las comunidades locales a quién ellas impactan. En palabras del autor: "[...] en este contexto de contradicciones implícitas dentro de un proyecto de integración regional sudamericano, nos corresponde cuestionar qué tipo de integración se está consolidando [...]" (OLIVEIRA, 2010, p. 44, traducción nuestra)².

Es decir, al proponer la integración y facilitar el flujo de mercancías, las rutas bioceánicas no solo están impactando la economía latinoamericana, sino en la vida y cultura de aquellas poblaciones cuyos territorios serán atravesados por estos proyectos. Es desde esta perspectiva que se expone aquí la historia del Corredor Vial Bioceánico - Brasil, Paraguay, Argentina y Chile - Ruta Porto Murtinho - Puertos del Norte de Chile, cuyo inicio se remonta a 2015, cuando los jefes de Estado de Brasil, Paraguay, Argentina y Chile firmaron un acuerdo para la integración vial en la agenda.

Según sus proponentes, el Corredor crea una importante conexión vial entre el Medio Oeste brasileño y el Océano Pacífico, cruzando los municipios de Porto Murtinho (Brasil); Carmelo Peralta (Paraguay), Mariscal (Paraguay), Estigarribia (Paraguay), Pozo Hondo (Paraguay), Misión La Paz (Argentina), Tartagal (Argentina), Jujuy (Argentina), Salta (Argentina) y Passo de Jama (Chile) hasta llegar a los puertos de Antofagasta, Mejillones e Iquique (Chile). Así, el trabajo debe reducir el tiempo de desplazamiento de la carga, mejorar la logística del transporte y aumentar la competitividad de las exportaciones a Asia, así como fomentar el turismo, estimular la creación de nuevos flujos comerciales regionales y profundizar la integración entre estos países (ALMEIDA; TEIXEIRA; FIGUEIRA, 2019).

Entre las acciones políticas para la ejecución del proyecto, los presidentes de los países involucrados aprobaron, en 2015, la Declaración de Asunción, documento que dio origen a un grupo de trabajo, cuyo propósito fue realizar estudios técnicos sobre la viabilidad del trabajo antes mencionado. Este grupo fue formado por el Ministerio de Transporte, Puertos y Aviación Civil y está bajo la coordinación de las cancillerías de los cuatro países involucrados. Además, formó parte de varias otras instituciones vinculadas al comercio exterior y al área de logística (MATO GROSSO DO SUL, s.d.).

En convergencia con este proyecto más amplio, en junio de 2016 se tomó otra acción política para viabilizar la integración en la agenda. En este caso, Brasil y Paraguay acordaron construir un puente sobre el río Paraguay, que unirá los municipios de Porto Murtinho, del lado brasileño, y Carmelo Peralta, del lado paraguayo ${ }^{3}$. De conformidad con el Acuerdo, la materialización del puente se basa sobre los principios de igualdad de derechos y deberes, en la preocupación por las cuestiones ambientales, económicas y sociales.

Por lo tanto, está claro que desde su inicio el proyecto se ha preocupado por los impactos sociales del trabajo en las comunidades locales. La medición de estos impactos, así como las propuestas de acciones para mitigarlos, han sido una preocupación del proyecto en su conjunto, por lo que es necesario tanto relevar estos posibles efectos en la ubicación como - con base en esta información - la preparación de planes de acción. Además, dicha información es necesaria para subsidiar también proyectos de desarrollo social local.

\footnotetext{
2 "[...] neste contexto de contradições implícitas dentro de um projeto de integração regional sul-americana, cabenos o questionamento de que tipo de integração está sendo consolidada [...]" (OLIVEIRA, 2010, p. 44).

${ }^{3}$ El Acuerdo para la construcción del puente fue aprobado mediante Proyecto de Decreto Legislativo n. 17, de 2018 (n. 709 de 2017 en la Cámara de Diputados), publicado en el Diario Oficial del Senado Federal el 14 de marzo de 2018 (BRASIL, 2018).
} 
Es precisamente en este contexto que, en julio de 2016, aparece la Red Universitaria del Corredor Bioceánico (UniRila)" ${ }^{4}$ entre cuyos objetivos se encuentran "[...] la realización de un inventario de investigaciones y publicaciones de las Universidades integradas al Corredor, la elaboración de una base de datos centralizada y formulación de proyectos que tengan como objetivo promover el desarrollo social" (ALMEIDA; TEIXEIRA; FIGUEIRA, 2019, p. 287).

En este sentido, la Universidad juega un papel fundamental de articulación entre las entidades gestoras y la comunidad local, por lo que UniRila nació, específicamente, un mes después de la firma del Convenio para la construcción del puente Brasil-Paraguay, a partir de un encuentro entre los Ministerio de Relaciones Exteriores, Gobierno del Estado de Mato Grosso do Sul y la Universidad Estatal de Mato Grosso do Sul (UEMS), en el ámbito del Grupo de Trabajo dedicado al estudio de viabilidad del proyecto. La acción se desarrolló durante el Seminario “Corredor Vial Bioceánico 'Brasil, Paraguay y Chile'/Ruta: Porto-Murtinho - Puertos del Norte de Chile", que se llevó a cabo en la ciudad de Campo Grande, Mato Grosso do Sul (MS), y en el que se expusieron y debatieron temas ${ }^{5}$ que implicaran la implementación del Corredor. En ese momento, entonces, se decidió, a través de la Carta de Campo Grande, que la UEMS coordinaría las iniciativas de apoyo de UniRila en Brasil (MATO GROSSO DO SUL, s.d.).

La Red Universitaria también identificó, en el ámbito del I Seminario de la Red Universitaria de la Ruta de la Integración Latinoamericana, en 2017, tres grandes ejes fundamentales para el desarrollo de la labor académica, a saber: I) Internacionalización y movilidad académica; II) Desarrollo local y turístico y III) Impactos sociales (ALMEIDA, TEIXEIRA, FIGUEIRA, 2019, p. 288).

A partir de estos ejes temáticos principales, se organizaron subgrupos de trabajo, entre ellos el Grupo de Trabajo de Educación, dentro del cual se está desarrollando la investigación que originó este estudio. Así, los grupos de trabajo de UniRila han colaborado con la implementación del Corredor Bioceánico, considerando que, según Weber (2008), las universidades no basan la investigación solo en temas académicos, sino que ingresan al área social, dedicándose a la actualidad sin perder conocimiento científico autónomo.

\section{EL MUNICIPIO BRASILEÑO DE PORTO MURTINHO: POBLACIÓN Y ASPECTOS ECONÓMICOS}

Porto Murtinho es uno de los 588 municipios brasileños en el territorio fronterizo internacional y uno de los 122 que son limítrofes. Esta franja recorre $15.719 \mathrm{~km}$ de territorio brasileño, llega a 11 Unidades de la Federación y alberga una población de unos 10 millones de habitantes, según el Instituto de Pesquisa Econômica Aplicada [IPEA] (2017). De hecho, el país tiene una inmensa zona fronteriza, siendo la tercera más larga del mundo, solo por detrás de Rusia y China (OLIVEIRA; MORELLO, 2019, p. 54).

Según la división política, la ciudad de Porto Murtinho se encuentra precisamente en la denominada "franja fronteriza", es decir, en la región que comprende el espacio que va desde la

\footnotetext{
${ }^{4}$ La sigla UniRila se justifica por el hecho de que el Corredor Bioceânico inicialmente se denominó Ruta de la Integración Latinoamericana (RILA).

${ }^{5}$ Entre los temas tratados se encuentran: la plataforma de gobernanza del Corredor Vial Bioceánico; la Declaración de Asunción, Comercio en Mesoamérica y Plataformas Logísticas; Tratados de Libre Comercio, Puente del Corredor Bioceánico, Papel de la Aduana en el Corredor, Impacto Socioeconómico del Corredor Bioceánico en la Región e Infraestructura Portuaria y Logística del Norte de Chile, entre otros.
} 
línea divisoria entre los países hasta $150 \mathrm{~km}$ hacia el interior del país. territorio nacional, siendo, por tanto, una de las 32 ciudades gemelas ${ }^{6}$ de Brasil (IPEA, 2017).

Estar en la franja fronteriza implica características que hacen únicas a estas regiones, convirtiéndolas en espacios de intercambio cultural, especialmente en el caso de las fronteras capilares y sinápticas ${ }^{7}$. Según Raffestin (2005, p. 13, traducción nuestra) $)^{8}$ “[...] la frontera es uno de los elementos de la comunicación biosocial que asume una función reguladora. Es la expresión de un equilibrio dinámico que no solo se encuentra en el sistema territorial, sino en todos los sistemas biosociales".

De hecho, más que puntos fijos de división, los espacios que confluyen en la frontera son lugares de intercambio y convergencia, dado que la definición de límites territoriales es más que nada una decisión para el ejercicio del poder de los Estados Nacionales. Después de todo, la región natural (Ilamada Pantanal, en Brasil, y Chaco, en Paraguay), por ejemplo, no "respeta" las fronteras de estos estados, ocupando un área que los trasciende y mostrando que las convergencias naturales y culturales de estos las regiones, de estos pueblos, son más intensas de lo que pueden suponer los límites políticos. En este caso, la frontera actúa como otro aspecto complejante de la dinámica local, en la gestión de políticas sociales, económicas y lingüísticas, por ejemplo.

Desde el punto de vista político-económico, si bien existen regiones fronterizas con flujo intenso (ver el caso de Ponta Porã, por ejemplo), existen otras como las regiones de Arcos Norte y Central, siendo esta última la que comprende el Selva Amazonia y Pantanal Sul-Matogrossense (región de Corumbá y Porto Murtinho), que están menos habitados en comparación con el Arco Sur del país, y también están mal integrados al mercado, desde el punto de vista económico (OLIVEIRA; MORELLO, 2019). Este hecho se destaca al pensar en la ciudad de Porto Murtinho, que solo recientemente ha recibido una mayor visibilidad, con la implementación del Corredor Bioceánico. De hecho, es un municipio pequeño, con poco más de quince mil habitantes, según datos del Instituto Brasileño de Geografía y Estadística (IBGE, 2010).

Ubicado en el Pantanal Sul-Mato-grossense, limítrofe con Paraguay, Porto Murtinho se caracterizó históricamente - en términos de economía local - por la actividad agrícola y la exploración del quebracho, del cual se extrae el tanino, habiendo vivido también un período próspero con el ciclo de la yerba. Además de estos, extrae parte de los ingresos de la exploración minera de piedra caliza y de la actividad portuaria fluvial presente desde su asentamiento (MATO GROSSO DO SUL, 2019, p. 1).

\footnotetext{
${ }^{6}$ Las ciudades gemelas son aquellas en las que el territorio del municipio limita con el país vecino y su sede está ubicada en la frontera internacional, que puede tener o no una conurbación o semi-conurbación con ubicación en el país vecino (NÚCLEO ESTADUAL PARA O DESENVOLVIMENTO E INTEGRAÇÃO DA FAIXA DE FRONTEIRA DE RONDÔNIA [NEINFRO], s.d.).

${ }^{7}$ Las interacciones fronterizas de tipo capilar son aquellas que ocurren "[...] solo a nivel local, como en el caso de las ferias, ejemplo concreto de interacción e integración fronteriza espontánea. Puede ocurrir a través de intercambios difusos entre vecinos con redes de comunicación limitadas, o resultar de zonas de integración espontánea, en las que el Estado interviene poco, principalmente al no invertir en la construcción de infraestructura de articulación transfronteriza". Por otro lado, "[...] el modelo Synapse se refiere a la presencia de un alto grado de intercambio entre poblaciones fronterizas sustentadas por Estados contiguos. Las ciudades gemelas más dinámicas se pueden caracterizar de acuerdo con este modelo". (NEIFRO, s.d.).

8 "[...] a fronteira é um dos elementos da comunicação biossocial que assume uma função reguladora. Ela é a expressão de um equilíbrio dinâmico que não se encontra somente no sistema territorial, mas em todos os sistemas biossociais" (RAFFESTIN, 2005, p. 13).
} 
La población de Porto Murtinho estuvo ligada a la vocación portuaria de la región que se encuentra a orillas del río Paraguay, colindante con el país homónimo. Creado inicialmente como Distrito de la ciudad de Corumbá, Mato Grosso do Sul, Porto Murtinho fue emancipado en 1912, mediante Decreto Estatal n. 310.

Con poco más de cien años de historia, el último censo de población realizado en 2010 por el IBGE contaba con una población de 15.372 habitantes, de los cuales 437 son extranjeros, lo que representa alrededor del 2,8\% de la población, que está compuesta mayoritariamente por personas entre 10 y 49 años (IBGE, 2010). Aproximadamente un tercio de la población vive en zonas rurales y el resto en zonas urbanas, según indicadores proporcionados por la Secretaría de Estado de Medio Ambiente, Desarrollo Económico, Producción y Agricultura Familiar (SEMAGRO) (MATO GROSSO DO SUL, 2019).

Esta población no encuentra suficientes trabajos en el municipio. Los indicadores mostraron que solo el 7.7\% de la población estaba ocupada económicamente en 2017 (IBGE, 2017). A la baja tasa de ocupación se suma la mala distribución de los ingresos. Si bien los datos del indicador del IBGE muestran un ingreso mensual promedio en el municipio de 2.3 salarios mínimos, el porcentaje de la población con un ingreso mensual nominal per cápita de hasta medio salario mínimo es de 40.4\%. Este hecho colocó al municipio, en 2017, en el puesto 15 en términos de distribución del ingreso en el Estado de Mato Grosso do Sul, que cuenta con setenta y nueve municipios (IBGE, 2017).

Los indicadores para el año 2019, proporcionados por SEMAGRO, destacan que las principales actividades económicas del municipio son la ganadería y el comercio. Destaca la ganadería, seguida por porcinos, equinos, ovinos y avícolas, además de sus productos: lana, leche, miel de abejas y huevos de gallina. En agricultura, las principales producciones son arroz, caña de azúcar, frijol, mandioca, maíz y soja. También está la exploración mineral de cobre, arena y granito para revestimiento (MATO GROSSO DO SUL, 2019).

El comercio, la segunda actividad que más ocupa la población de Porto Murtinho, es relativamente pequeña, con 163 establecimientos en 2018, entre ellos un mayorista y los demás minoristas. Ese mismo año, la ciudad solo contaba con nueve industrias, dos en el área de la construcción civil; uno en el área de alimentos; tres para la preparación del cuero y sus artefactos y otros tres clasificados como varios. En cuanto a la prestación de servicios, el municipio contaba con veintisiete establecimientos (MATO GROSSO DO SUL, 2019).

Estos indicadores llevan a cuestionar las posibilidades de empleo de jóvenes y adultos en el municipio, dado el reducido número de establecimientos comerciales, industriales y de prestación de servicios; los dos últimos, en su mayoría, orientados a satisfacer las necesidades de la producción agrícola local (MATO GROSSO DO SUL, 2019). Este escenario puede cambiar con la implementación del Corredor Bioceánico, ya que agilizará el flujo de mercancías en la región, ya sea por el puente vial Porto Murtinho-Carmelo Peralta o por los puertos de lluvia instalados en la ciudad.

De hecho, la construcción del puente ya está modificando Porto Murtinho, que se ha convertido en el objetivo de interés de los mercados asiáticos. Además, se están construyendo tres nuevos puertos de tormenta en el municipio con la perspectiva de un cuarto puerto. Sumando los recursos privados asignados en los puertos, los del estado de Mato Grosso do Sul y los de la Unión en infraestructura, se invertirán en el municipio alrededor de 650 millones de reales, de los cuales se destinarán a la ampliación y mantenimiento de BR 267. Organizando el ingreso y salida de carga de estos puertos, en 2020 se construyó un estacionamiento de camiones a veinte 
kilómetros de la ciudad, con el objetivo de contener el flujo y permanencia de estos vehículos en el casco urbano del municipio (ANDRADE, 2019).

Todos estos cambios han impactado en la región de Porto Murtinho y deberían replicarse a lo largo del Corredor Bioceánico, en diferentes contextos locales. Como muestran los indicadores, el municipio en cuestión es pequeño, con poca oferta de servicios y comercio y una población que, al no encontrar trabajo, suele emplearse en otras localidades. En este sentido, la implementación del Corredor tenderá a crear nuevos puestos de trabajo en la ciudad (construcción civil, servicios, mayor turismo local, etc.), manteniendo y también ampliando su población.

En consecuencia, también aumentará la demanda de bienes sociales como vivienda, salud y educación. En el caso de estos últimos, el impacto no solo debería estar en el número de plazas en las escuelas regulares, sino también en los tipos y niveles de formación para el trabajo, como cursos técnicos y de calificación, por ejemplo; así como con respecto a la calificación de maestros y otros trabajadores de la educación. Así que echemos un vistazo más de cerca al panorama educativo actual en la ciudad.

\section{EL MUNICIPIO BRASILEÑO DE PORTO MURTINHO: ASPECTOS DEL CONTEXTO EDUCATIVO ACTUAL}

Comprender la oferta educativa actual en las escuelas públicas de Porto Murtinho es fundamental para que en los próximos años sea posible medir los impactos en la educación en el municipio debido a la implementación del Corredor Bioceánico, así como los impactos de las medidas de planificación previstas. de tales cambios.

En este contexto, en lo que respecta al acceso a la educación escolar, el municipio tiene una tasa de escolarización del 94,1\% para niños y adolescentes entre 6 y 14 años, público objetivo de la Educación Primaria, que es una de las etapas obligatorias de la escolarización en Brasil. En 2020, las 12 escuelas públicas de la ciudad atendieron a un total de 3.462 estudiantes, divididos entre jardín de infantes, primaria y secundaria. De estas 12 escuelas, según el catálogo de escuelas del INEP, 11 son municipales y 1 son estatales, con 9 en el área urbana y 3 en el área rural, y de estas últimas, 2 están ubicadas en tierras indígenas.

En cuanto a las etapas de enseñanza ofrecidas, la única escuela estatal ofrece educación primaria, secundaria y educación para jóvenes y adultos. Las escuelas municipales se dividen de la siguiente manera: 3 ofrecen sólo jardín de infancia; 1 solo escuela primaria; 1 solo educación de jóvenes y adultos; 1 escuela primaria y educación de jóvenes y adultos; 1 jardín de infantes y escuela primaria; 3 ofrecen educación infantil, primaria y educación de jóvenes y adultos, y la última que no informó las etapas atendidas (INEP, 2020). Por tanto, más de la mitad de las escuelas de Porto Murtinho ofrecen dos o más etapas educativas, en las que las matrículas se distribuyen de la siguiente manera:

Tabla 1 - Matrículas en el municipio de Porto Murtinho-MS por etapas de Educación Básica

\begin{tabular}{|c|c|c|c|c|c|c|c|c|c|}
\hline Total & \multicolumn{3}{|c|}{ Educación Infantil } & \multicolumn{3}{c|}{ Enseñanza Fundamental } & \multicolumn{3}{c|}{ Escuela Secundaria } \\
\hline & Total & Guardería & Preescolar & Total & $\begin{array}{c}\text { Primeros } \\
\text { Años }\end{array}$ & $\begin{array}{c}\text { Últimos } \\
\text { años }\end{array}$ & Total & Propedéutico & Técnico \\
\hline 2019 & 580 & 271 & 309 & 2.492 & 1.524 & 968 & 505 & 505 & 0 \\
\hline 2020 & 584 & 240 & 344 & 2.351 & 1.372 & 979 & 527 & 527 & 0 \\
\hline
\end{tabular}

Fuente: INEP (2020). 
Los indicadores anteriores muestran que los primeros años de Educación Primaria son la mayor demanda educativa en Porto Murtinho, incluso con una caída de alrededor de 150 matrículas en esta etapa entre 2019 y 2020. Otros puntos que llaman la atención son I) el bajo número de estudiantes matriculados en el bachillerato y II) la falta de provisión de educación profesional a nivel de bachillerato, lo que podría ser problematizado por las bajas tasas de empleabilidad y el reducido número de industrias en la ciudad.

Además, estos indicadores apuntan al incumplimiento de una de las metas establecidas en el Plan de Desarrollo e Integración de la Franja Fronteriza/MS, publicado en 2012 por el Gobierno del Estado de Mato Grosso do Sul. Según el Plan, la educación es un eje estratégico, asumiendo como meta en este contexto "[...] incrementar el número de cursos técnicos en educación profesional para los jóvenes residentes en los municipios fronterizos" (MATO GROSSO DO SUL, 2012, p. 4, traducción nuestra) $)^{9}$. Parece que, dada la implementación de la Ruta Bioceánica, tanto el Estado como el sector privado con interés en el desarrollo local tienen, entonces, ante sí la urgente tarea de inversión local e implementación de este tipo de cursos, a riesgo de atrayendo únicamente mano de obra especializada temporal de otros lugares sin necesariamente invertir en la calificación de la comunidad local, por ejemplo. La inversión en la calificación local, por lo tanto, es una condición necesaria para que el proyecto Rota tenga un impacto positivo en la comunidad local.

Además, la tabla anterior también muestra una disminución importante en el número de inscripciones en la escuela secundaria en relación con la escuela primaria (calificaciones finales), cuyas causas aún deben ser profundamente investigadas por esta investigación. Sin embargo, a pesar de que esta disminución solo sigue una tendencia nacional en este nivel de educación, la oferta de mayores oportunidades de calificación e ingresos a los jóvenes de la localidad del grupo de edad que comprende el bachillerato, derivada de la expansión de la empleabilidad a los calificados. el trabajo, por ejemplo, podría centrarse en este contexto de abandono de la escuela secundaria. En este aspecto, se debe tomar una acción estratégica hacia la expansión de la educación local tanto por el Estado y Municipio como por el sector privado involucrado en la planificación y ejecución de la Ruta. En cuanto a las demás modalidades de Educación Básica, tenemos la siguiente situación:

Tabla 2 - Matrículas en el municipio de Porto Murtinho-MS por modalidades de Educación Básica

\begin{tabular}{|c|c|c|c|c|c|c|}
\hline & \multicolumn{3}{|c|}{ Educación de jóvenes y adultos } & \multicolumn{3}{c|}{ Educación especial } \\
\hline Año & Total & $\begin{array}{c}\text { Enseñanza } \\
\text { fundamental }\end{array}$ & $\begin{array}{c}\text { Escuela } \\
\text { secundaria }\end{array}$ & Total & $\begin{array}{c}\text { Clases } \\
\text { comunes }\end{array}$ & $\begin{array}{c}\text { Clases } \\
\text { exclusivas }\end{array}$ \\
\hline 2019 & 324 & 254 & 70 & 85 & 73 & 12 \\
\hline 2020 & 302 & 232 & 70 & 77 & 60 & 17 \\
\hline
\end{tabular}

Fuente: INEP (2020).

Aún con la tasa de escolaridad superando el 94\%, la participación de la Educación de Jóvenes y Adultos, que está "[...] destinada a quienes no tuvieron acceso o continuidad de estudios en

\footnotetext{
9 "[...] elevar o número de cursos técnicos da educação profissional para os jovens residentes nos municípios da faixa de fronteira" (MATO GROSSO DO SUL, 2012, p. 40).
} 
la educación primaria y secundaria a su edad" (BRASIL, 1996, p. 30, traducción nuestra) ${ }^{10}$, es bastante significativo, ya que representa aproximadamente el 10,2\% de los estudiantes que asisten a la escuela primaria y el $14 \%$ de los que asisten a la escuela secundaria. Indicadores que nos dan indicios de que la tasa de escolaridad ha avanzado en un período reciente, justificando la permanencia de esta medida educativa compensatoria.

En cuanto a la Educación Especial, "[...] tipo de educación escolar, preferentemente ofrecida en el sistema escolar regular, para estudiantes con necesidades especiales" (BRASIL, 1996, p. 39, traducción nuestra), resultante de deficiencias, desórdenes globales o altas capacidades, entre 2019 y 2020, el municipio de Porto Murtinho atendió a alrededor de 80 estudiantes, el público objetivo de este tipo de educación. La mayoría de ellos fueron atendidos en salas comunes de escuelas regulares, en cumplimiento de la Ley de Lineamientos y Bases de la Educación Nacional (BRASIL, 1996).

Para atender la demanda educativa del municipio en 2019, se necesitaron 219 docentes. En el año siguiente, 211 docentes trabajaron en las diferentes etapas de la docencia, como se muestra en la siguiente tabla:

Tabla 3 - Número cuantitativo de docentes que trabajan en el municipio de Porto Murtinho-MS por etapas de Educación Básica

\begin{tabular}{|c|c|c|c|c|c|c|c|c|c|}
\hline & \multicolumn{3}{|c|}{ Educación Infantil } & \multicolumn{3}{c|}{ Enseñanza fundamental } & \multicolumn{3}{c|}{ Escuela secundaria } \\
\hline Año & Total & Guardería & Preescolar & Total & $\begin{array}{c}\text { Primeros } \\
\text { años }\end{array}$ & $\begin{array}{c}\text { Últimos } \\
\text { años }\end{array}$ & Total & Propedéutico & Técnico \\
\hline 2019 & 68 & 30 & 38 & 164 & 83 & 110 & 45 & 45 & 0 \\
\hline 2020 & 57 & 26 & 33 & 159 & 103 & 93 & 41 & 41 & 0 \\
\hline
\end{tabular}

Fuente: INEP (2020).

Así, los indicadores muestran que parte de los docentes laboran en más de una etapa de la docencia, ya que la suma de docentes que les dedican supera al total de docentes que laboran en las redes docentes en los años respectivos.

En cuanto a los niveles de certificación docente, tenemos la siguiente tabla para el año 2020:

Tabla 4 - Niveles de certificación docente en el municipio de Porto Murtinho-MS en 2020

\begin{tabular}{|c|c|c|c|c|c|c|c|}
\hline \multicolumn{2}{|c|}{} & Sin graduación & \multicolumn{2}{c|}{ Graduados } & \multicolumn{3}{c|}{ Postgraduado } \\
\hline & Total & Escuela secundaria & Licenciatura & Sin licenciatura & Especialización & Maestría & Doctorado \\
\hline N & 211 & 42 & 165 & 4 & 84 & 0 & 0 \\
\hline$\%$ & 100 & $19,9 \%$ & $78,2 \%$ & $1,90 \%$ & $39,8 \%$ & $0 \%$ & $0 \%$ \\
\hline
\end{tabular}

Fuente: INEP (2020).

Como se muestra en los datos anteriores, de los poco más de 200 docentes que trabajaron en las redes de Educación Estatal y Municipal en Porto Murtinho, en 2020, alrededor del 80\% eran graduados, y la mitad de ellos tenían un título de especialista. Sin embargo, llama la atención la ausencia total de profesores con títulos de maestría o doctorado. Se requieren estudios más

10 "[...] destinada àqueles que não tiveram acesso ou continuidade de estudos no ensino fundamental e médio na idade própria" (BRASIL, 1996, p. 30). 
detallados al respecto, que deberán ser tratados en futuras fases de esta investigación, junto con otros aspectos como el análisis de la presencia y repercusión de las políticas públicas federales en conjunto con los estados y municipios para la formación continua de docentes, que ejemplos son los programas de Maestría Académica Profesional.

Aún en la formación docente, se observa la persistencia de casi el 20\% de los docentes con solo educación secundaria, lo que - a pesar de estar respaldado legalmente por la Ley de Lineamientos y Bases de la Educación Nacional, n. 9.394/96 (BRASIL, 1996) - demuestra cierto estancamiento en el proceso de calificación de quienes educan.

En cuanto al régimen de contratación o tipo de relación laboral en las diferentes redes de educación pública, los profesores que trabajan en Porto Murtinho vivieron las siguientes situaciones en 2020:

Tabla 5 - Régimen de contratación o tipo de vínculo y Dependencia Administrativa de los docentes del municipio de Porto Murtinho-MS-2020

\begin{tabular}{|c|c|c|c|c|c|}
\hline \multicolumn{2}{|c|}{} & \multicolumn{2}{|c|}{ Examen público eficaz / estable } & \multicolumn{2}{c|}{ Contrato provisional $^{\mathbf{1 1}}$} \\
\hline & $\begin{array}{c}\text { Total de Contratos } \\
\text { en el Municipio }\end{array}$ & Red Estatal & Red Municipal & Rede Estatal & Red Municipal \\
\hline $\mathrm{N}$ & $249^{12}$ & 4 & 56 & 43 & 146 \\
\hline$\%$ & 100 & $1,6 \%$ & $22,48 \%$ & $17,26 \%$ & $58,63 \%$ \\
\hline
\end{tabular}

Fuente: INEP (2020).

Los indicadores presentados apuntan a un hecho alarmante: menos del $30 \%$ de los contratos se encuentran en licitación pública efectiva/estable en 2020; los demás son contratos temporales, lo que es un indicador de precariedad tanto en el proceso educativo como en las condiciones laborales docentes.

Con base en la realidad de la Red de Educación del Estado en el Estado de São Paulo, por ejemplo, Basílio y Almeida (2018) aclaran que el ingreso a la carrera docente mediante examen público garantiza la estabilidad (despido por proceso administrativo); régimen especial de jubilación y acceso a prestaciones como la asistencia sanitaria, además de un plan de carrera que permite la progresión funcional tanto por antigüedad como por titulación.

Además, desde el punto de vista pedagógico, la estabilidad en una institución específica contribuye a la construcción de un sentido de pertenencia e identificación con esa comunidad escolar, lo que a su vez contribuye a que se convierta en una comunidad de práctica (WENGER, 1998), posibilitando el surgimiento de proyectos educativos a largo plazo, por ejemplo. Esto se ve muy afectado cuando un docente cambia con frecuencia de una institución a otra, dado el contexto temporal y, por lo tanto, bastante incierto de su régimen de trabajo.

Además, en cuanto a la precariedad de sus condiciones laborales, los docentes no titulares enfrentan numerosas situaciones personalmente negativas, como contratos de duración determinada con percepción de ingresos referidos únicamente a las horas de clases impartidas;

\footnotetext{
${ }^{11}$ En 2020, las redes de enseñanza de Porto Murtinho no contaban con docentes subcontratados o docentes bajo el régimen CLT- Consolidação das Leis Trabalhistas (INEP, 2020).

12 Existe una discrepancia en los datos entre el número de profesores y el número de contratos, ya que un mismo profesor puede tener más de un (tipo) de contrato en (más de) una Red Educativa.
} 
contratos ocasionales de menos de quince días para el suministro de licencias sanitarias para profesores permanentes, que no permiten predecir cuánto se recibirá a fin de mes; no hay garantía de derechos como el cuidado de la salud; cumplimiento de la brecha entre contratos y consecuentes diferencias en relación a la retribución y la seguridad social (BASÍLIO; ALMEIDA, 2018).

La situación de precariedad expuesta anteriormente incorpora a los docentes que ocuparon alrededor del $70 \%$ de los contratos temporales en Porto Murtinho en 2020. Las políticas que establecen o mantienen este tipo de precariedad están claramente alineadas con la perspectiva neoliberal, priorizando un llamado uso racional de los recursos públicos. con miras a reducir el gasto del Estado en servicios sociales esenciales, como la educación, específicamente porque la contratación temporal abarata el costo de la mano de obra (GENTILI, 1996). En este caso, el desarrollo socioeconómico real de la región no se da, debido a la contención de recursos cuyos propósitos, en general, pueden no beneficiar a la comunidad.

Esta precariedad obviamente afecta la calidad de la educación que se practica en las escuelas. Así lo apuntan los propios instrumentos de evaluación a gran escala, propuestos por la misma lógica neoliberal (GENTILI, 1996). En este contexto, destacamos el Índice de Desarrollo de la Educación Básica (IDEB), creado por el INEP en 2007, y que se calcula a partir de las categorías aprendizaje y flujo, el primero medido mediante el desempeño de los estudiantes en el Prova Brasil y el Sistema de Evaluación de la Educación Básica (SAEB), y el segundo a través de la medición de las tasas de aprobación y reprobación de los estudiantes.

Conscientes de las contradicciones y limitaciones en el uso del IDEB como instrumento para evaluar la calidad de la educación brasileña, pero también conscientes de que han sido ampliamente aceptadas como parámetro para la calidad de la educación, los resultados de la Red Pública del municipio de Porto Murtinho a partir de $4^{\circ}$ grado $/ 5^{\circ}$ grado y $8^{\circ}$ grado $/ 9^{\circ}$ grado:

Tabla 6 - IDEB Porto Murtinho-MS- 2005-2017

\begin{tabular}{|c|c|c|c|c|c|c|c|c|}
\hline & $2005^{13}$ & 2007 & 2009 & 2011 & 2013 & 2015 & 2017 \\
\hline \multirow{2}{*}{ 4a grado / 5 año } & Resultados & 3.1 & 3.5 & 3.8 & 4.2 & 4.6 & 4.6 & 4.8 \\
\cline { 2 - 9 } & Objetivo & - & 3.1 & 3.5 & 3.9 & 4.2 & 4.5 & 4.8 \\
\hline \multirow{2}{*}{ 8a grado / 9 año } & Resultados & 3.3 & 3.5 & 3.7 & 3.7 & 3.7 & 4.0 & 4.4 \\
\cline { 2 - 10 } & Objetivo & - & 3.3 & 3.4 & 3.7 & 4.1 & 4.5 & 4.8 \\
\hline
\end{tabular}

Fuente: INEP (2018).

Los indicadores anteriores muestran un mejor desempeño de los alumnos en los primeros años de la Escuela Primaria, ya que los de cuarto/quinto grado superaron la meta entre 2005 y 2015 y la alcanzaron en 2017. Los octavos/noveno grados, a su vez, aunque presentaron incrementos IDEB, no alcanzaron las metas fijadas para los años 2013, 2015 y 2017. Dichos resultados, por lo tanto, apuntan a la necesidad de mejoras en la oferta educativa del municipio. Pese a ello, es necesario advertir una vez más que, como advierten Oliveira y Araújo (2005, p. 18),

[...] los indicadores de desempeño están prácticamente inexplorados en la realidad brasileña, ya que los sistemas educativos no cuentan con metodologías para evaluarlos. [...] Así, las pruebas estandarizadas son instrumentos necesarios, pero insuficientes para mejorar la calidad de la enseñanza. Además, existen cuestiones metodológicas que dificultan el análisis

\footnotetext{
${ }_{13}$ Embora o IDEB tenha sido criado em 2007, o SAEB já era aplicado desde a década de 1990, o que permite o cálculo do índice anterior à sua criação oficial para fins de comparação.
} 
de los impactos que los insumos educativos, el contexto socioeconómico y cultural y las prácticas escolares tienen en el aprendizaje de los estudiantes. (OLIVEIRA; ARAÚJO, 2005, p. 18, traducción nuestra) $)^{14}$.

En este sentido, aunque pueden ser considerados como proveedores de datos relevantes, es necesario considerar en los análisis educativos las limitaciones de las pruebas estandarizadas y del propio IDEB, ya que responden a la necesidad de control por parte de los gobiernos neoliberales, que descentralizan las acciones educativas (como como el proceso de municipalización), sino que centralizan la medición de los resultados de estas acciones y poco (o nada) hacen con ellas, dada la dificultad de analizar cualitativamente los distintos elementos que inciden en el proceso de enseñanza-aprendizaje, que no se limitan únicamente a la contexto escolar. Esta pregunta también justifica la presente investigación, que buscará, en fases posteriores, precisamente un análisis más profundo de las escuelas de Porto Murtinho.

En este caso, por ejemplo, analizaremos, además de las repercusiones de los aspectos socioeconómicos discutidos hasta ahora, también un aspecto de gran relevancia en contextos fronterizos, que es el intercambio de estudiantes entre municipios vecinos, en el caso de Carmelo Peralta (Paraguay). Al respecto, Silva y Moreira Junior (2019, p. 1115, traducción nuestra) ${ }^{15}$ aclaran que:

[...] la escuela de la frontera presenta, de manera acentuada, esta pluralidad cultural, reuniendo a estudiantes que no solo tienen diferentes culturas, costumbres, tradiciones y realidades, sino que muchas veces también hablan un idioma diferente. Ante esto, el trabajo del docente se torna aún más desafiante, pues le corresponde a él proveer que brasileños y extranjeros, en este caso los paraguayos, tengan las mismas condiciones de aprendizaje y desarrollo.

Para ello, Silva y Moreira Junior (2019, p. 1117, traducción nuestra) advierten que “[...] es necesario que los departamentos de educación (estatales o municipales) creen proyectos y estrategias para que la educación de frontera pueda, de hecho, ofrecer un servicio educativo significativo a los estudiantes paraguayos que se matriculan en escuelas brasileñas". Específicamente sobre la relación Brasil-Paraguay, Silva y Moreira Junior (2019) explican que la oferta educativa

[...] está establecido de tal manera que Brasil asume la responsabilidad, en gran parte de su frontera con Paraguay, de la escolarización de los estudiantes fronterizos que residen dentro de ciertos límites territoriales, considerando que la educación está constituida en la Constitución Federal Brasileña. derecho subjetivo y deber del Estado. Sin embargo, muchos paraguayos estudian en escuelas ubicadas en el lado brasileño de la frontera. Es importante resaltar que se trata de paraguayos que tienen doble ciudadanía, lo que les da derecho a

\footnotetext{
14 [...] os indicadores de desempenho são praticamente inexplorados na realidade brasileira, pois os sistemas de ensino não possuem metodologias para avaliá-los. [...] Dessa forma, os testes padronizados são instrumentos necessários, mas insuficientes para a melhoria da qualidade de ensino. Ademais, há questões de ordem metodológica que dificultam a análise dos impactos que os insumos educacionais, o contexto socioeconômico e cultural e as práticas escolares têm sobre a aprendizagem dos alunos (OLIVEIRA; ARAÚJO, 2005, p. 18).

15 [...] a escola na fronteira apresenta, de forma acentuada, essa pluralidade cultural, reunindo alunos que têm não somente culturas, costumes, tradições e realidades distintas, mas muitas vezes também falam uma língua diferente. Diante disso, o trabalho do professor trona-se ainda mais desafiador, pois cabe a ele proporcionar que brasileiros e estrangeiros, no caso aqui os paraguaios, tenham iguais condições de aprendizagem e desenvolvimento (SILVA; MOREIRA JUNIOR, 2019, p. 1115).
} 
Ios servicios sociales de uso colectivo (SILVA; MOREIRA JUNIOR, 2019, p. 1114, traducción nuestra $)^{16}$.

Por lo tanto, atender a estudiantes paraguayos con doble ciudadanía es una práctica común en las escuelas de municipios fronterizos como Porto Murtinho. Sin embargo, el mapeo de este servicio requiere una investigación in situ, ya que, como aclaran Silva y Moreira Junior (2019), el Censo Escolar realizado por el INEP no identifica el número de estudiantes paraguayos atendidos en las escuelas brasileñas en las regiones fronterizas ni siquiera la situación de aprendizaje en consideración del plurilingüismo/plurilingüismo, por ejemplo, que constituye estas regiones. Este es un punto que merece una mirada más atenta, que será dada por esta investigación, dentro de lo que se pueda discutir, incluyendo los proyectos de las Escuelas Bilingües de Fronteira, por ejemplo. Pero este es un punto que quedará para las próximas fases de la investigación y para otras publicaciones.

\section{CONSIDERACIONES FINALES}

Los indicadores poblacionales, económicos y educativos aquí presentados, los análisis y discusiones realizados son el resultado de los esfuerzos iniciales para comprender la realidad social, económica y educativa del municipio de Porto Murtinho, colindante con el municipio paraguayo de Carmelo Peralta. Es un hecho que tal situación fronteriza complejiza y enriquece la localidad en lo que respecta al intercambio cultural, la variedad lingüística y, en consecuencia, las demandas sociales como la educación.

Con poco más de quince mil habitantes y cuatro mil alumnos atendidos por doce escuelas públicas, Porto Murtinho ya se caracteriza por la diversidad cultural y lingüística dada la presencia de poblaciones indígenas y habitantes extranjeros. Por tanto, se cree que la implementación del Corredor Bioceánico se enfocará en un contexto que en sí mismo es diverso en términos culturales y lingüísticos y tenderá a incrementar la demanda de educación escolar ya que puede impulsar la permanencia de habitantes que hoy no encuentran puestos de trabajo en el municipio y también la llegada de nuevos sujetos, dado el potencial crecimiento económico que representa la implementación del Corredor Bioceánico.

Concluimos, con base en lo anterior, que, para mitigar los posibles impactos negativos, el crecimiento económico debe ir acompañado de la expansión de las inversiones en educación, de cara a superar problemas ya identificados, como la insuficiencia de los concursos públicos para la contratación de docentes; ampliación de las unidades escolares, que actualmente se dividen en ofrecer más de una etapa de educación básica; ofreciendo formación profesional de bachillerato y la creación y fortalecimiento de políticas orientadas a atender a estudiantes paraguayos y brasileños en una perspectiva multi/multicultural.

\footnotetext{
${ }^{16}$ [...] é estabelecida de forma que o Brasil assume a responsabilidade, em grande parte de sua fronteira com o Paraguai, pela escolarização dos alunos fronteiriços residentes dentro de determinados limites territoriais, tendo em vista que na Constituição Federal brasileira a educação se constitui um direito subjetivo e um dever do Estado. Muitos paraguaios, no entanto, estudam em escolas situadas do lado brasileiro da fronteira. É importante destacar que se trata de paraguaios que possuem dupla cidadania, o que lhes confere o direito aos serviços sociais de uso coletivo (SILVA; MOREIRA JUNIOR, 2019, p. 1114).
} 


\section{REFERENCIAS}

ALMEIDA, Luciane Pinho; TEIXEIRA, Léia Lacerda; FIGUEIRA, Kátia Cristina Nascimento. A importância do estudo dos impactos sociais junto às comunidades locais dos territórios que integram o Corredor Rodoviário Bioceânico. Interações, Campo Grande, v. 20, número especial, p. 285-96, 2019. Disponible en: http:// www.scielo.br/scielo.php?script=sci_arttext\&pid=S1518-70122019000500285 Acceso el: 30 mar. 2020.

ANDRADE, Silvio. Obras de infraestrutura para nova rota comercial começam a transformar Porto Murtinho. Portal do Governo de Mato Grosso do Sul, [s.l.], 2019. Disponible en: http://www.ms.gov.br/obras-deinfraestrutura-para-nova-rota-comercial-comecam-a-transformar-porto-murtinho/. Acceso el: 24 ago. 2020.

BRASIL. Projeto de Decreto Legislativo n. 17 de 2018 (n. 709/2017). Aprova o texto do acordo entre o Governo da República Federativa do Brasil e o Governo da República do Paraguai para a construção de uma ponte rodoviária internacional sobre o rio Paraguai entre as cidades de Porto Murtinho e Carmelo Peralta. Brasília, DF, 2018. Disponible en: https://legis.senado.leg.br/sdleg-getter/documento?dm=7644 691\&disposition=inline. Acceso el: 16 abr. 2020.

BRASIL. Lei n. 9.394, de 20 de dezembro de 1996. Estabelece as diretrizes e bases da educação nacional. Diário Oficial da União, Brasília, DF, 20 dez. 1996. Disponible en: https://www2.senado.leg.br/bdsf/ bitstream/handle/id/529732/lei_de_diretrizes_e_bases_1ed.pdfhttp://www.planalto.gov.br/ccivil_03/ leis/I9394.htm. Acceso el: 15 feb. 2017.

BASILIO, Juliana Regina; ALMEIDA, Ana Maria Fonseca. Contratos de trabalho de professores e resultados escolares. Revista Brasileira de Educação, [s.I.], v. 23, e230049, 2018.

GAMBOA, Silvio Sánchez. Quantidade-qualidade: para além de um dualismo técnico e de uma dicotomia epistemológica. In: GAMBOA, Silvio Sánchez; SANTOS FILHO, Camilo (Org.). Pesquisa educacional: quantidade-qualidade. 7. ed. São Paulo: Cortez, 2009. p. 84-110. (Coleção Questões da Nossa Época, v. 42).

GENTILI, Pablo Antonio Amadeo. Neoliberalismo e educação: manual do usuário. In: SILVA, Tomaz Tadeu; GENTILI, Pablo A. A. (Org.). Escola S. A.: quem ganha e quem perda no mercado educacional do neoliberalismo. Brasília, DF: CNTE, 1996. p. 9-49.

IBGE. Porto Murtinho: trabalho e rendimento. IBGE, Brasília, DF, 2017. Disponible en: https://cidades. ibge.gov.br/brasil/ms/porto-murtinho/panorama. Acceso el: 19 de abr. 2020.

IBGE. Censo 2010. IBGE, Brasília, DF, 2010. Disponible en: https://censo2010.ibge.gov.br/. Acceso el: 19 abr. 2020.

INSTITUTO NACIONAL DE ESTUDOS E PESQUISAS EDUCACIONAIS ANÍSIO TEIXEIRA [INEP]. Sinopses estatísticas do censo escolar 2020. INEP, Brasília, DF, 2021. Disponible en: http://portal.inep.gov.br/ censo-escolar. Acceso el: 11 mayo 2021.

INSTITUTO NACIONAL DE ESTUDOS E PESQUISAS EDUCACIONAIS ANÍSIO TEIXEIRA [INEP]. Catálogo de escolas. INEP, Brasília, DF, 2020. Disponible en: https://www.gov.br/inep/pt-br/acesso-a-informacao/ dados-abertos/inep-data/catalogo-de-escolas. Acceso el: 10 feb. 2021.

INSTITUTO NACIONAL DE ESTUDOS E PESQUISAS EDUCACIONAIS ANÍSIO TEIXEIRA [INEP]. Sinopses estatísticas do censo escolar 2019. INEP, Brasília, DF, 2019. Disponible en: http://portal.inep.gov.br/ censo-escolar. Acceso el: 22 abr. 2020.

INSTITUTO NACIONAL DE ESTUDOS E PESQUISAS EDUCACIONAIS ANÍSIO TEIXEIRA [INEP]. IDEB Resultados e metas: Porto Murtinho, MS. INEP, Brasília, DF, 2018. Disponible en: http://ideb.inep.gov.br/resultado/ resultado/resultado.seam?cid=448775. Acceso el: 7 mayo 2020. 
INSTITUTO DE PESQUISA ECONÔMICA APLICADA [IPEA]. Faixa de Fronteira do Brasil é tema de estudo do Ministério da Integração e Ipea. IPEA, Brasília, DF, 2017. Disponible en: https://bit.ly/2YfaMcK. Acceso el: 10 feb. 2021.

MATO GROSSO DO SUL (Estado). Secretaria de Estado de Meio Ambiente, Desenvolvimento Econômico, Produção e Agricultura familiar. Coordenadoria de Economia e Estatística. Dados Estatísticos dos Municípios de MS: Porto Murtinho, 2019. Campo Grande: SEMAGRO, 2019. Disponible en: http://www.semagro. ms.gov.br/perfis-socioeconomicos-do-ms-e-municipios/. Acceso el: 6 mayo 2020.

MATO GROSSO DO SUL (Estado). Secretaria do Estado de Meio Ambiente, do Planejamento, da Ciência e Tecnologia. Secretaria de Estado de Desenvolvimento Agrário, da Produção, da Indústria, do Comércio e do Turismo. Plano de Desenvolvimento e Integração da Faixa de Fronteira/MS. [S.I.]: Governo do Estado de Mato Grosso do Sul, 2012. Disponible en: http://www.semagro.ms.gov.br/wp-content/uploads/2017/06/ Plano-Desenvolvimento-e-Integra\%C3\%A7\%C3\%A3o-de-Fronteira.pdf. Acceso el: 10 jun. 2021.

MATO GROSSO DO SUL (Estado). Antecedentes. Corredor Bioceânico, [s.I.], [s.d.]. Disponible en: http:// www.corredorbioceanico.ms.gov.br/antecedentes/. Acceso el: 30 mar. 2020.

NÚCLEO ESTADUAL PARA O DESENVOLVIMENTO E INTEGRAÇÃO DA FAIXA DE FRONTEIRA DE RONDÔNIA [NEIFRO]. A criação dos núcleos estaduais de fronteira. Neifro, Porto Velho, [s.d.]. Disponible en: http:// www.neifro.ro.gov.br/Conteudo/Exibir/43. Acceso el: 29 abr. 2020.

NÚCLEO ESTADUAL PARA O DESENVOLVIMENTO E INTEGRAÇÃO DA FAIXA DE FRONTEIRA DE RONDÔNIA [NEIFRO]. Cidades-gêmeas. Neifro, Porto Velho, [s.d.]. Disponible en: http://www.neifro.ro.gov.br/ Conteudo/Exibir/43. Acceso el: 29 abr. 2020.

OLIVEIRA, Gilvan Muller; MORELLO, Rosângela. A fronteira como recurso: o bilinguismo portuguêsespanhol e o projeto escolas interculturais bilíngues de fronteira do MERCOSUL (2005-2016). Revista Iberoamericana de Educación, Madrid, v. 81, n. 1, p. 53-74, 2019.

OLIVEIRA, Romualdo Portela; ARAÚJO, Gilda Cardoso. Qualidade do ensino: uma nova dimensão da luta pelo direito à educação. Revista Brasileira de Educação, [s.l.], n. 28, p. 5-24, 2005. Disponible en: http:// www.scielo.br/pdf/rbedu/n28/a02n28.pdf. Acceso el: 10 jun. 2016.

OLIVEIRA, Valquíria de Araújo. A infraestrutura de transportes como política governamental para o desenvolvimento regional e a integração sul-americana: uma análise sobre as Rotas Bioceânicas em Mato Grosso do Sul. 2010. 145 f. Dissertação (Mestrado em Geografía) - Universidade Federal de Grande Dourados, Dourados, MS, 2010.

RAFFESTIN, Claude. Prefácio. In: OLIVEIRA, Tito Carlos Machado (Org.). Território sem limites. Campo Grande: UFSM, 2005.

REDE UNIVERSITÁRIA DA ROTA DE INTEGRAÇÃO LATINO-AMERICANA [UNIRILA]. Termo de convênio de cooperação recíproca, de 22 ago. 2019. Campo Grande: UniRila, 2019.

SILVA, Rodrigo Rocha; MOREIRA JUNIOR, Orlando. A educação na fronteira entre Brasil e Paraguai: narrativas (auto) biográficas de um fronteiriço. Revista Brasileira de Pesquisa (Auto) Biográfica, Salvador, v. 4, n. 12, p. 1110-24, 2019. Disponible en: https://www.revistas.uneb.br/index.php/rbpab/article/view/5894. Acceso el: 20 jun. 2021.

WEBER, M. Economía y sociedad: esbozo de sociología comprensiva. Cidade do México: Fondo de Cultura Económica, 2008.

WENGER, Etienne. Communities of practice. Cambridge: Cambridge University Press, 1998. 


\section{Sobre las autoras:}

Jaqueline Basso: Doctora y máster en Educación por la Universidad Federal de São Carlos (UFSCar). Grado en Pedagogía por la Universidad Brasil (UB). Profesora adjunta de la Universidad Estatal de Mato Grosso do Sul (UEMS), Unidad Campo Grande. Profesora titular del programa de posgrado Maestría Profesional en Educación de la UEMS. Investigadora del Grupo de Estudio e Investigación en Educación Rural de la UFSCar y del Grupo Trabajo, Educación y Políticas Públicas de la UEMS. Coordinadora del GT Educación del eje Impactos Sociales de UniRila. Membresía: UEMS. E-mail: jaqueline.basso@uems.br, Orcid: https://orcid.org/0000-0002-4262-4140

Vanessa Arlésia de Souza Ferretti: Doctora y máster en Lingüística por la Universidad Federal de Santa Catarina (UFSC). Grado en Letras por la Universidad Estatal de Río de Janeiro (UERJ). Profesora adjunta de la Universidad Estatal de Mato Grosso do Sul (UEMS). Coordinadora del Curso de Licenciatura en Letras Portugués-Español y sus Literaturas, de la UEMS, Unidad Campo Grande. Integra el Núcleo de Estudios Bakhtinianos (NEBA) y el grupo de investigación Educación, Cultura y Diversidad, vinculado a la Red Internacional de Investigación para América Latina, Europa y el Caribe/Red ALEC. Editora de la web Revista Linguagem, Educação e Memória. Realiza investigaciones basadas en Estudios Críticos del Discurso y Análisis Dialógica del Discurso. E-mail: vanessa.ferretti@uems.br, Orcid: https://orcid.org/0000-0003-1245-4301 